\title{
Yuánmíng Yuán en el siglo XVIII: Arte entre la diplomacia y la filosofía; entre Europa y Pekín
}

\section{Yuánmíng Yuán in the 18th Century: Art between Diplomacy and Pilosophy; between Europe and Pekin}

\author{
Pedro Luengo ${ }^{1}$ \\ Universidad de Sevilla (España)
}

Recibido: 23-12-15

Aprobado: 21-01-16

\section{Resumen}

Este estudio pretende probar que el Yuánmíng Yuán fue diseñado como una representación del macrocosmos imperial a partir de la filosofía de la China de Qianlong. Para ello se valorará la función simbólica de los palacios de estilo europeo a partir de las descripciones de embajadas orientales y occidentales. Esta información se pondrá en relación con las diferentes representaciones pictóricas del jardín, conservadas tanto en grabados o acuarelas como en pinturas murales. A partir de toda esta información se pretende valorar su utilización en las visitas de diferentes legados diplomáticos extranjeros. En concreto, se intenta comprobar que los palacios europeos se utilizaron eminentemente para impresionar a las misiones orientales, y no tanto a las occidentales.

Palabras-clave: Jesuitas en China, Jardín chino, Palacios barrocos, híbridos, diálogo cultural.

\begin{abstract}
This study aims to prove that the Yuánmíng Yuán was designed as a representation of the imperial macrocosm from the philosophy of Qianlong China. To do so, the symbolic role of European-style palaces from descriptions

\footnotetext{
${ }^{1}$ (pedroluengo@us.es). Contrato de Acceso al Sistema Español de Ciencia de la Universidad de Sevilla. Universidad de Sevilla. Doctor en Historia del Arte. Recientemente ha publicado Intramuros, arquitectura en Manila 1739-1762. Madrid, Fundación Española de Universidades, 2012 y Manila, plaza fuerte. Ingenieros militares entre Europa, América y Asia. Madrid, Ministerio de Defensa, 2014. El presente trabajo forma parte del proyecto Identidad europea y arquitectura globalizada en el Pekín de Qianlong (HAR2014-61714-EXP).
} 
of Eastern and Western embassies. This information will be enriched with the various pictorial representations of the garden, conserved in both watercolors, prints or murals. From all this information is intended to assess its use in the visits of different foreign legacies. Specifically, we try to ensure that European palaces were used predominantly to impress the Eastern missions, rather than the Western.

Key-words: Jesuits in China, Chinese garden, Baroque palaces, hybrids, cultural dialogue.

Los estudios sobre el jardín-palacio del Yuánmíng Yuán 圆明园 en los últimos años se han multiplicado, abordando cuestiones de diseño arquitectónico ${ }^{2}$ o de patrimonialización, siendo mucho menor el interés que ha suscitado su uso original ${ }^{3}$. Aunque es conocida su vinculación con el emperador y la corte, poco se ha escrito sobre la función habitual de estos edificios, especialmente exóticos dentro de la producción china del momento ${ }^{4}$, resultando más atractiva la faceta de transferencia técnica de la construcción ${ }^{5}$.

${ }^{2}$ Desde principios del siglo XX existió un interés sobre este híbrido arquitectónico, como demuestran trabajos como el de Maurice Adam, Yuen Ming Yuen. L'Oeuvre Architecturale des Anciens Jésuites au XVIIIe siècle, Pekin, Imprimerie des lazaristes, 1936; o bien el de Hope Danby, The Garden of Perfect Brightness: the history of the Yüan Ming Yüan and of the emperors who lived there, Chicago, Williams and Norgate, 1950. A finales del siglo XX, el interés se retomó gracias a trabajos como el de Michèle Pirazzoli-t'Serstevens (ed.), Le Yuanmingyuan. Jeux d'eau et palais européens du XVIIIe siècle à la cour de Chine, Paris, ERC, 1987. Mucho más numerosos son los trabajos del siglo XXI centrados en el jardín completo: Che Bing Chiu, Un grand jardin imperial chinois: le Yuanming yuan, jardin de la Clarté parfait, "Extrême-Orient, Extrême-Occident", 22 (2000), pp. 17-50; Daiheng Guo, 圆明 园的”记忆遗产” 样式房图档. Hangzhou: Zhejiang gu ji chu ban she, 2010; Daiheng Guo (ed.), 远 逝的辉煌: 圆明园建筑园林研究与保护, Shanghai, Shanghai keji jishu chubanshe, 2009. Esto debe complementarse con los trabajos específicos sobre los edificios europeos: Geremie R. Barmé. The Garden of Perfect Brightness, a life in ruins, "East Asian History", 11 (1996), pp. 111-158. Greg. M. Thomas, Yuanming Yuan/Versailles: Intercultural Interactions between Chinese and European Palace Cultures, "Art History", 32/1 (2009), pp. 115-143; Hui Zou, A Jesuit Garden in Beijing and Early Modern Chinese Culture. West Lafayette, Purdue University Press, 2011. Zheng Yangwen, An Unexplained Image from the Garden of Perfect Brightness, "China Heritage Quarterly”, 29 (2012). Qingyu Jiang. Yuanming Yuan Garden: Space Arrangement Principles among Italy and China in Eighteenth Century. PhD Thesis. Politecnico di Torino, 2014, por citar solo algunos.

3 Victoria M. Cha-Tsu Siu. Gardens of a Chinese Emperor. Imperial Creations of the Qianlong Era, 1736-1796. Bethlehem, Lehigh university press, 2013. Kristina Kleugthen, Imperial Illusions: Crossing Pictorial Boundaries in the Qing Palaces, Seattle: University of Washington Press, 2015.

${ }^{4}$ A los edificios de este jardín habría que incluir las iglesias levantadas en la capital. Algunos de las aproximaciones más recientes a este tema son: Pedro Luengo. Arquitectura jesuita en Filipinas y China [en María Isabel Álvaro Zamora, Javier Ibáñez Fernández y Jesús Criado Mainar (coord.), La arquitectura jesuítica, Zaragoza, Institución Fernando el Católico, 2012], pp. 523-540; o Pedro Luengo. Identidad y globalización en las fachadas jesuitas de Pekín en el siglo XVIII' [en María Isabel Álvaro Zamora y Javier Ibáñez Fernández (coords.), La Compañía de Jesús y las artes: nuevas perspectivas de investigación, Zaragoza, Universidad de Zaragoza, 2014], pp. 279-300.

${ }^{5}$ Benjamin A. Elman. The Jesuit Role as “Experts” in High Qing Cartography and Technology. “臺大歷史學報National Taiwan University History Bulletin”, 31 (2003), pp. 223-250. 
Este trabajo pretende demostrar que el jardín tenía como principal objetivo revelar al emisario extranjero las maravillas arquitectónicas más lejanas a su tradición, insistiendo en el carácter central y universal del imperio chino. En cambio, aquellas construcciones que debían resultarles más cercanas, no se les mostraban y quedaban escondidas en la inmensidad del jardín 6 . Para probar esta hipótesis se utilizarán varias descripciones conocidas de misiones diplomáticas de diferente procedencia realizadas durante el siglo XVIII: desde la inglesa hasta la holandesa pasando por otras orientales. Sus descripciones se ubicarán dentro del jardín, localizando los espacios que debieron conocer. Por otro lado, las series de grabados y acuarelas del jardín conservadas serán relacionadas con la información de las descripciones, intentando explicar por qué ninguna de ellas incluye puntos de todo el recinto.

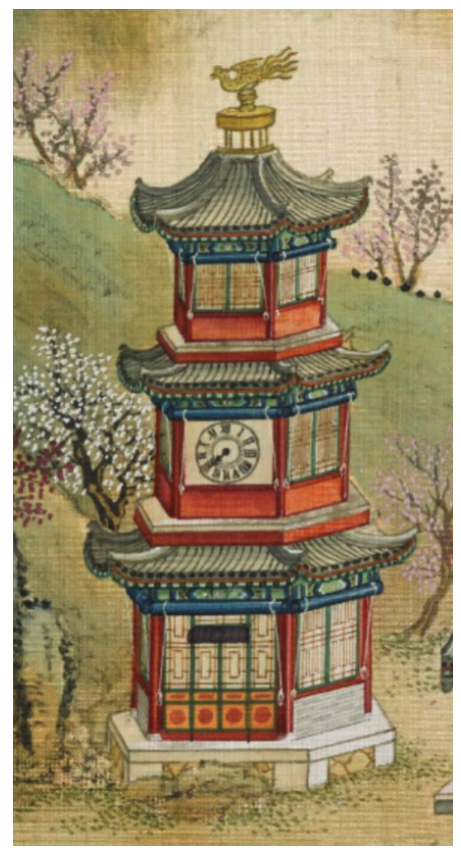

Fig. 1. Detalle del reloj occidental en la pagoda. Imagen tomada de 海淀: 中国皇帝的行宫.

\footnotetext{
${ }^{6}$ Una descripción del funcionamiento del jardín queda manifestada en "Mais au moins qu'on pose en principe dans nos mœurs, si l'on veut encore en avoir, que tout jardin public demande pour la conservation de la décence et le maintien du bon ordre, une distribution ouverte, spacieuse et telle que le plus habile architecte des jardins, le célèbre le Nostre, les a tracés et exécutés, en variant toujours ses plans sur le terrain qu'on lui donnait". Louis-François Delatour, Essais sur l'architecture des Chinois sur leurs jardins, leurs principes de médecine et leurs moeurs et usages. Paris, Clousier, 1803, p. 154.
} 


\section{Diseño del Yuánmíng Yuán}

Este Jardín-Palacio es el primer ejemplo de esta tipología en China, tras las transformaciones de Yōngzhèng 雍正, ya como emperador en $1725^{7}$. Sería su sucesor, Qianlong (1735-1796), quien durante su largo gobierno rediseñara el jardín-palacio. Aunque los jardines imperiales habían servido desde antiguo como residencias temporales, no estaban diseñados para alojar a la corte durante la mayor parte del año. A partir del modelo de Yuánmíng Yuán aparecerían otros como el Bìshǔ shānzhuāng 避暑山庄 en Rehe o el posterior Yíhéyuán 䝠和园 en Pekín. Las nuevas funciones del jardín obligaron a planificarlo en consonancia con la simbología adecuada alrededor del lago Hòuhú 后湖. Así el jardín fue concebido como un microcosmos de todo el imperio chino, con el emperador en el centro y las nueve divisiones a su alrededor (Jiuzhou 九州) $)^{8}$. Aunque todas las zonas participaban de la universalidad del imperio (Fig. 1), algunas subrayaban más claramente una tradición específica. De hecho uno de los espacios del Yuánmíng Yuán (Fig. 2), el Jiǔzhōu qīng yàn 九州清晏, es decir Paz y Tranquilidad en las nueve divisiones, hace referencia a esta interpretación? 9 La organización espacial basada en el nueve, con el emperador en el centro del diagrama, forma parte de la tradición china desde época antigua ${ }^{10}$. El perímetro defensivo también emulaba el imperio, con forma de abanico y protección hacia tres lados: oeste, norte y este. El sur quedaba protegido por el mar. Así, quedaban fuera los templos Wàibāmiào 外 八庙, que imitaban técnicas constructivas propias de zonas fuera del imperio. En este contexto es más sencillo de explicar la presencia de una concubina uigur en la corte ${ }^{11}$. La representación de un amplio espectro cultural no se hacía por tanto con la arquitectura en exclusividad, sino por propuestas culturales paralelas. Si la arquitectura intentaba hacer presente los estilos propios de tradiciones distantes, la naturaleza estaba diseñada de un modo similar. Así, en el jardín podían encontrarse especies propias del ámbito siamés, entre otras.

Algunos de los edificios vinculados con la vida del emperador (Jiǔzhōu qīng yàn, 九州清宴) y la administración del imperio (Qín zhèng qīn xián, 勤政親賢) se

\footnotetext{
${ }^{7}$ Para designar este modelo en el que los pueblos y los lugares del imperio quedaban representados en el jardín-palacio, se ha aceptado el término Lígōng xíng huángjiā yuánlín 離宮型皇家园林. Véase Cary Y. Liu. Archive of Power. The Qing Dinasty Imperial Garden Palace at Rehe. “國立臺灣大學 美術史研究集刊”, 28 (2010), p. 44.

${ }^{8}$ El emperador quedó en el centro del lago representado por el pabellón de audiencias mientras que las divisiones fueron representadas por las nueve islas del lago. Las nueve direcciones o Jiuzhou九州 quedan mencionada en el capítulo Yugong 禹貢 del Shujing o bien Shangshu 尚书. Geremie R. Barmé. Op. cit., p.117.

${ }^{9}$ Cary Y. Liu. Architects and Builders of the Qing Dynasty Yuanming Yuan Imperial GardenPalace, "The University of Hong Kong Museum Journal", 1 (2002), pp. 38-161.

${ }^{10}$ John B. Henderson. Chinese Cosmographical Thought: The High Intellectual Tradition [en J. B. Harley and David Woodward (ed.), Cartography in the Traditional East and Southeast Asian Societies, Chicago, The University of Chicago Press, 1994], pp. 203-227.

${ }^{11}$ James A. Millward, A Uyghur Muslim in Qianlong's Court. The Meaning of the Fragant Concubine, "The Journal of Asian Studies", 53/2 (1994), pp. 427-458.
} 
encontraban frente al acceso al complejo (Zhèngdà guāngmíng, 正大光明) y de espaldas al lago Hòuhú 后湖 (Fig. 2) ${ }^{12}$. En la orilla del lago se desarrollaban algunos complejos que recordaban arquitecturas del imperio, tales como el Shàngxià tiānguāng 上下天光 basado en edificios de la provincia de Hunan, pero no es el único caso. Otro ejemplo lo ofrece el Guòrán dàgōng 廓然大公 que emula el famoso jardín del Jìchàng Yuán 寄畅 园 de Wuxi. También cabe destacar la Zuòshí línliú 坐石臨流, que recuerda una calle comercial de Suzhou. Todo esto puede vincularse con el hecho de que el emperador había realizado viajes a las provincias del sur, inspección conocida como Nán xún 南巡. Durante este tiempo pudo conocer personalmente algunos de los jardines más famosos, del mismo modo que hizo anteriormente su antecesor Kangxi. Una de las más de cuarenta recreaciones que se han identificado en todo el Yuánmíng Yuán referidas a tradiciones del sur del imperio, es el Jardín de Ānlán 安瀾園, ubicado originalmente en la casa de la familia Chén 陳氏 en Haining 海寧 ${ }^{13}$. Algo similar ocurre con la biblioteca Tiān yī gé 天一閣 de la familia Fàn 范氏 en Ningbo 宁波; y con el Jardín del león 獅子林 de Suzhou ${ }^{14}$. También en este lago se construyó el Qīngyànfãng 清晏舫 (1775), barco de mármol que incorpora referencias decorativas propias del mundo mogol (Fig. 2). Aunque la intención general era ambientar una zona concreta del territorio imperial, no era raro encontrar referencias exóticas dentro del planteamiento, como es el reloj occidental incorporado en una de las torres de este último complejo constructivo (Fig. 1).

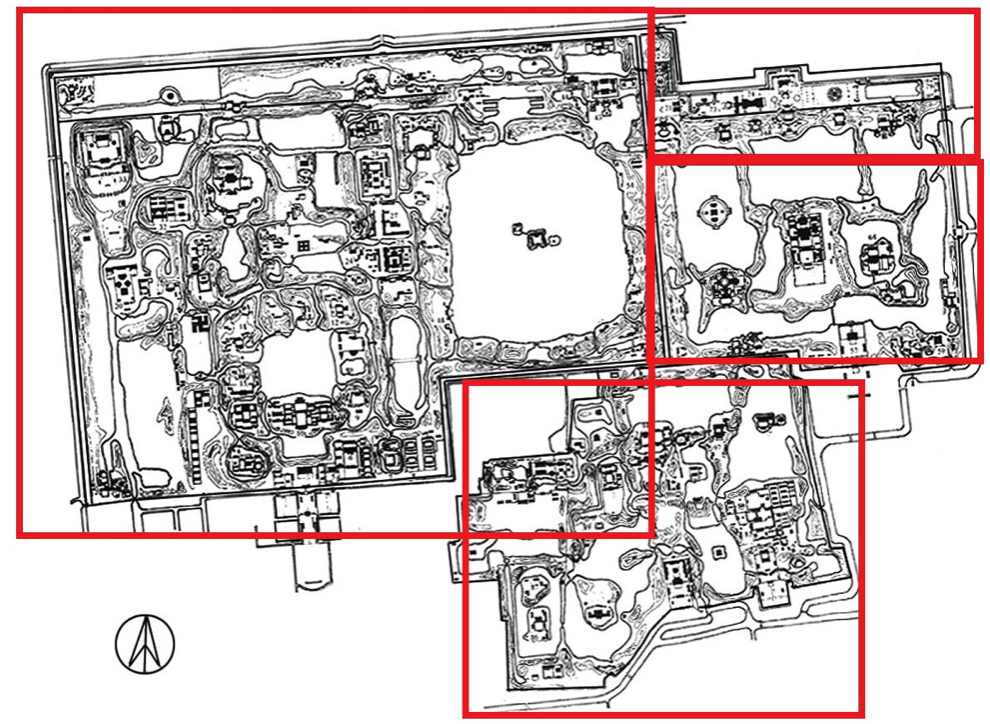

Fig. 2. China. Pekín. Jardín del Yuánmíng Yuán. De izquierda a derecha y de arriba abajo: Yuánmíng Yuán 圆明园, Xīyánglóu 西 洋楼, Qǐchūn yuán绮春园 y Chăngchūn yuán长春园.

${ }^{12}$ Para más información sobre la vida diaria en el jardín véase Victoria M. Cha-Tsu Siu. Op. cit.

13 Geremie R. Barmé. Op. cit., p. 121.

14 Ibid., p. 121. 
En el jardín no sólo se encontraban edificios que podrían catalogarse como recreativos o administrativos, sino que eran habituales los templos donde el emperador desarrollaba los diferentes rituales propios de varios credos: desde los tibetanos a los taoístas, por citar solo algunos ${ }^{15}$. Uno de los edificios más señalados de la actividad religiosa en el jardín tiene forma de esvástica (Fig. $2)^{16}$. Los cuatro brazos del edificio se consideraban representativos de las cuatro partes del mundo, que se unían en el centro. Otros de estos complejos reproducían arquitecturas budistas y taoístas como el Bìtóng shūyuàn碧桐 書院 o el Cíyún pǔhù 慈蓉普護. En un ámbito secundario con respecto al lago se encuentran otros edificios que emulan la arquitectura mongola, tales como el Dòngtiān shēn chù 洞天深處 o el Shāngāo shuǐ cháng 山高水长. Incluso el Rúān yòugōng 安佑宫位, también conocido como Hóngcí yǒnghù 鴻慈永祜, ofrece algunos elementos que pueden asociarse con la tradición mongola. Los tres estaban separados, en puntos muy distantes entre sí, pero todos ellos también separados del lago. Parece claro que el lago servía de centro referente como imagen del imperio, y las variaciones territoriales se disponen concéntricamente a este punto.

Pero a estos más conocidos se pueden unir también otros ejemplos como los templos tibetanos del Zhèng jué sì 正觉寺, en el extremo sur; el Hòu dà miào 后大庙 construido originalmente como un lamaserio de tres pisos; el Xūmí língjìng 须弥灵境 en la parte del Lago Kunming, vinculados ya con el Yíhé Yuán 㟲和园. Sin duda, el interés del emperador por las culturas más lejanas está detrás de estas construcciones, pero también un conocimiento profundo de estas. Con el caso tibetano, las relaciones son muy estrechas como ya han apuntados varios autores, lo que dio lugar a numerosas reconstrucciones de estructuras tibetanas preexistentes y a nuevas obras ${ }^{17}$. El exotismo no era entendido simplemente como un ejemplo de las fronteras del imperio, sino como una parte consustancial del sentido budista del emperador, como señor de los nueve espacios.

Esta complejidad, representados además pictóricamente en volúmenes de vistas, ha llevado a algunos especialistas a clasificar los espacios a partir de un esquema religioso por orden de importancia ${ }^{18}$.

\footnotetext{
${ }^{15}$ Ibid., pp. 18-19.

${ }^{16}$ Ibid., p. 24.

${ }_{17}$ Cabría destacar la reforma del palacio de Yonghe, el templo budista construido por Kangxi en Rehe, la reproducción de Potala o la residencia de los Pachen en Tashilhunpo, estas tres últimas dentro del mismo recinto. James Hevia, Lamas, Emperors, and Rituals: Political Implications in Qing Imperial Ceremonies. "Journal of the International Association of Buddhist Studies", 16/2 (1993), p. 251. Ver también Xiangyun Wang, The Qing Cout's Tibet Connection: Lcang skya Rol pa'i rdo rje and the Qianlong Emperor. "Harvard Journal of Asiatic Studies", 60/1 (2000), pp. 125-163.

${ }^{18}$ Liu Chang. From Study on Yuanming Yuan Interior Design to design Practice for the Beijing Chateau, Pekín, Tsinghua University, s.f., p. 24.
} 
Así se diferencian tres niveles: el mundano (incidiendo sobre diferentes aspectos del buen gobierno), el divino (incluyendo cuestiones religiosas) y el natural (incluyendo paisajes y representaciones rurales). Así, en aspectos religiosos se distinguen los espacios dedicados al taoísmo (Fānghú shèngjǐng, 方菛勝景 y Péngdăo yáotái 蓬島瑤台) y al budismo (Yuède Yúnjū 月地雲居 y Rì tiān Línyǔ 日天琳宇).

Las construcciones citadas se despliegan a lo largo del terreno conocido originalmente como Yuánmíng Yuán, que sería finalizado en 1744. Posteriormente se finalizarían otros dos jardines más: el Chăngchūn yuán 长春园 (1751) y el Qǐ chūn yuán 绮春园 (1772), que serían conocidos de forma general con el nombre del primero (Fig. 2). Otras estructuras propias de tradiciones extranjeras también se ubicaban en los márgenes del jardín. El ejemplo más claro lo ofrece la serie de palacios europeos o Xīyáng lóu 西洋楼 (Fig. 2), localizados en la esquina noreste de todo el jardín ${ }^{19}$. Se trata de uno de los extremos del recinto, justo tras un grupo de edificios que fueron descritos por Qiánlóng en el poema "El país de las maravillas en una maceta cuadrada". A partir de la leyenda a la que hace referencia, algunos autores han vinculado las montañas que se alejaban según se acercaba el visitante con la sección de los palacios europeos. Esta zona, que también incluía edificios de estética oriental tales como el Yăngquelóng 養隺籠 y el Zhútíng 竹亭 se levantó en dos fases entre $1747-1759^{20}$.

El mundo europeo debía suponer para Qiánlóng la parte más alejada del mundo conocido, lleno además de elementos maravillosos si bien difíciles de comprender (Fig. 3). Aunque los jesuitas jugaron un papel muy relevante a la hora de transmitir esta imagen al emperador, como ya ha sido apuntado por otros autores ${ }^{21}$, no fueron los únicos. Las diferentes embajadas procedentes tanto de países europeos tales como Portugal u Holanda, como la embajada papal o los envíos de Luis XIV pasando por los fondos que trasladaron a Pekín los misioneros de Propaganda Fide proporcionaron una biblioteca de contenidos visuales realmente destacable. El grado de actualización y el acceso a fuentes artísticas que tuvo Pekín en este momento es comparable a muchos centros secundarios dentro de la propia Europa.

La primera zona se inició en 1747, incluyendo el Xiéqíqù 谐奇趣, el Xùshuǐ lóu 蓄水楼, el Wánghuā zhèn 黃花陣y el citado Yăngquè lóng

\footnotetext{
${ }^{19}$ Hui Zou, The Jing of Line-Method: A perspective Garden in the Garden of Round Brightness, Montreal, McGill University, 2005, p. 203.

${ }^{20}$ Michèle Pirazzoli-t'Serstevens. Les Palais européens histoire et legends [en Michèle Pirazzolit'Serstevens (ed.), op. cit.], pp. 8-9.

${ }^{21}$ Zheng Yangwen, China on the Sea. How the Maritime World Shaped Modern China, Leiden, Brill, 2012 .
} 
养雀笼. Esta primera sección tiene una lógica plena, sin necesidad de las construcciones europeas posteriores. Así, cuando el emperador entraba en este "mundo maravilloso" necesitaba la protección desde el norte, por lo que se incluyó una construcción de estética china ${ }^{22}$. La segunda fase de construcción se iniciaría tras la terminación del Chăngchūn yuán 长春园 (Fig.2) en 1751, en torno a 1754, siendo terminado ya en 1759. Para unir las dos fases se diseñó una puerta con dos caras, una de estilo occidental y otra de tipo oriental ${ }^{23}$. Quedarían por incluirse en el complejo el edificio realizado para albergar los tapices enviados desde Francia, conocido como el Yuănyíngguān 远瀛观, iniciado en 1768. El emperador comenzaría entonces las nuevas obras con un palacio a la europea, para pasar después a un palacio a la italiana como indica concretamente Delatour ${ }^{24}$.

Con todos estos ejemplos se puede comprobar que la ubicación de los complejos arquitectónicos asociados a tradiciones ajenas al imperio quedaba fuera de los círculos inmediatos al centro de poder. Dicho esto, no existía un intento de traslación geográfica de la cartografía mundial a la organización del jardín imperial. Los edificios quedaban incluidos en el diseño, escondiéndose la mayor parte del tiempo a los visitantes. Esto, que resulta tan habitual en el mundo de Extremo Oriente, chocaba frontalmente con el diseño propio del jardín francés o italiano que se intentó emular en el Xīyánglóu 西洋楼 ${ }^{25}$. Esta diferencia tendría especial importancia en el diseño de los edificios europeos. Como complejo está oculto a la vista general del jardín. Ya en el interior, su compartimentación y diferente orientación, hacen imposible asimilarlo desde una única perspectiva como ocurría en otros jardines europeos. El juego con el visitante se mantuvo siguiendo el criterio oriental. Frente a esto, las líneas rectas y la organización pictórica del paisaje se mantuvieron fieles a una tradición europea en cualquier caso cercenada.

\footnotetext{
${ }^{22}$ Hui Zou. The Jing, p. 206.

${ }^{23}$ Ibid., pp. 207-208.

24 "La bâtisse de la première maison européenne ayant eu un plein succès, l'empereur désira une seconde maison, et on lui fit, pour varier ses jardins, un pavillon à l'italienne". Louis-François Delatour, op. cit., p. 152.

25 Mientras el Yuányě 園冶 de Jìchéng 計成 asegura que no importa que los caminos estén escondidos, siempre que estén diseñados de forma que giren y vuelvan (不妨偏 徑頓置婉轉), el prototipo occidental apuesta por una visión completa en un golpe de vista. Frente al concepto de "reemplazar el camino directo por el tortuoso" (以曲帶直), en Occidente se optó por la línea recta como patrón básico. Alison Hardie, trans., The Craft of Gardens, New Haven \& London, 1988.
} 


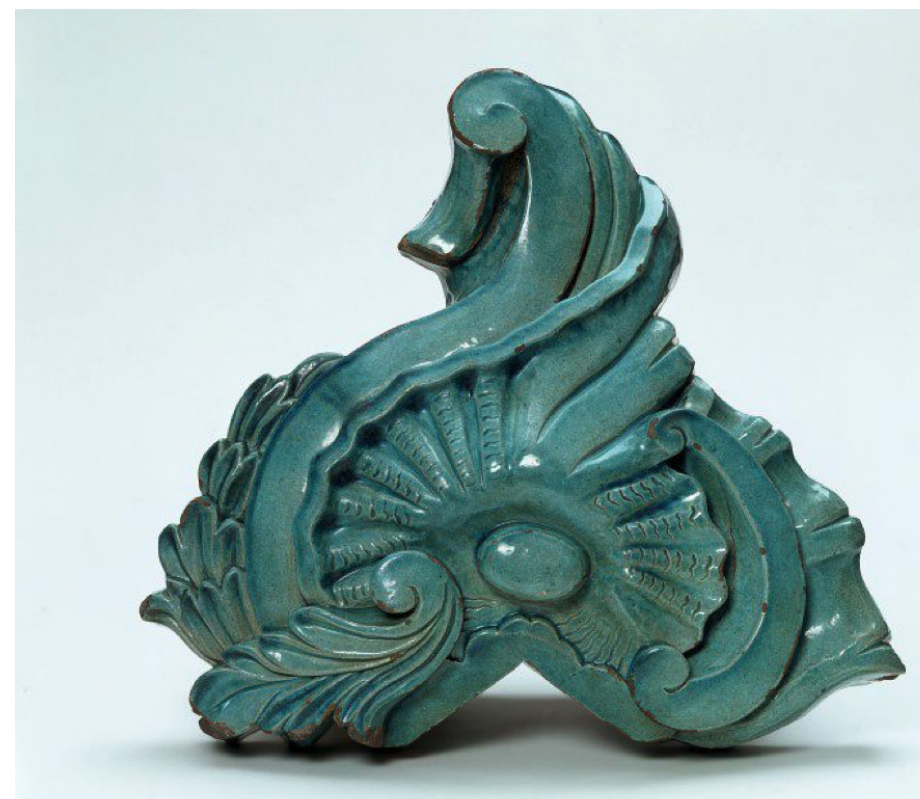

Fig. 3. Rocalla. Decoración vinculada con los restos de los palacios europeos del Yuánmíng Yuán de Pekín. Museo Victoria \& Albert, C.382-1912.

Tras esta breve explicación, resulta evidente que el imperio chino bajo la dinastía Qing fue capaz de plantear un modelo de jardín-palacio con aspiraciones universales, pero siguiendo un modelo muy diferente del europe ${ }^{26}$. Aunque eran conocedores de las obras de Versalles, incluso a través de los planos de Israel Silvestre, el modelo no se ajustaba a su concepto de relación con la naturaleza. De la misma forma, cabe ubicar la propuesta de Qiánlóng en paralelo con otras muchas contemporáneas entre las que se podrían citar el Topkapi Dîvân-1 Hümâyûn en Estanbul (Ahmet III, 1703-1736), el Chinesisches Haus de Sanssouci en Postdam (1755-1764), el Palacio Chino del Oranienbaum de San Petersburgo (1762-1768), incluso las Salas de Porcelana que introdujo Carlos III en el Palacio Real de Madrid y en el de Aranjuez (1765), y por último el pabellón chino de Versalles realizado en torno a 1776, así como otras consecuencias ligeramente más tardías en jardines británicos como Kew o Woburn Abbey, entre otros muchos. Todas ellas cuentan con referencias chinas, o europeas en el caso otomano, como elementos exóticos de un panorama general. Con excepción del caso español y el turco, su función dentro del jardín era muy limitada al recreo y no a la administración del imperio

${ }^{26}$ Esto ha sido planteado por diferentes estudios previos como Greg M. Thomas. Op. Cit., pp. 121-122. 
o al recibimiento de emisarios extranjeros. Así, aunque de una u otra forma las diferentes monarquías internacionales intentaron mostrarse ante sus cortes como universales, no todas lo hicieron de la misma forma.

\section{Descripciones las misiones diplomáticas del siglo XVIII}

Los jardines imperiales chinos han sido interpretados tradicionalmente como un lugar de esparcimiento cortesano con especial protagonismo del emperador. Como se ha apuntado, desde el siglo XVIII estos tomaron un nuevo sentido, convirtiéndose en espacios de diplomacia y gestión imperial. El espacio del jardín sería tomado entonces no sólo por los mandarines cortesanos, sino también por misiones extranjeras más o menos alejadas culturalmente del mundo chino. Esto ha permitido que sean varias las descripciones extranjeras del jardín, permitiendo una comparación hasta ahora inédita. Más concretamente, para este estudio se han utilizado varias descripciones del siglo XVIII. Se incluyen las de origen oriental: la del Pachen Erdeni ${ }^{27}$, una posible tibetana del $13^{\circ}$ Pachen Lama de $1780^{28}$ y una coreana ${ }^{29}$. Por desgracia, otras conocidas como la visita de los representantes del séptimo Dalai Lama (1708-1757) a Pekín en 1756, no cuentan con descripciones de los jardines ${ }^{30}$. A estas hay que unir además varias descripciones europeas: las inglesas de la embajada de George Staunton, George Macartney ${ }^{31}$ y John Barrow en 1792 $2^{32}$, la holandesa fruto de la embajada de Isaac Titsingh y Andreas Van Braam, de $1795^{33}$, y por último las descripciones de los misioneros franceses Attiret, Cibot $^{34}$ y Delatour, aunque no puedan considerarse misiones diplomáticas ${ }^{35}$.

${ }^{27}$ Aunque debió estar en China, su presencia en el Yuánmíng Yuán es aún difícil de confirmar, ya que sólo un misionero europeo, Amiot, afirma que estuvo en el jardín. Schuyler Cammann. The Panchen Lama's Visit to China in 1780: An Episode in Anglo-Tibetan Relations. "The Far Eastern Quarterly", 9/1 (1949), pp. 3-19. Siendo así, parece claro que estuvo ya que esta visita está descrita en una carta del propio emperador al Dalai Lama, traducida a su vez por Turner en su Embassy to Tibet. The Asiatic Anual Register. Londres, J. Debrett, 1801, pp. 70-71.

28 Anne Chayet, Une description tibétaine du Yuanmingyuan [en Michèle Pirazzoli-t'Serstevens. Le Yuanmingyuan..., ], p. 11.

${ }^{29}$ Citado en Young-tsu Wong. A Paradise lost: the imperial Garden Yuanming Yuan, Honolulu, University of Hawai'i Press, 2001, basándose en Yanxinglu xuanji (Selected travel notes from the Korean envoys to Beijing). Seúl: Dadong wenhua yanjiuso, 1961.

${ }^{30}$ Victoria M. Cha-Tsu Siu. Gardens..., p. 4.

31 George Staunton. Authentic Account of an Embassy from The King of Great Britain to the Emperor of China. Londres, W. Bulmer and Co., 1797.

32 John Barrow. Travels in China. Londres, Cadell and W. Davies, 1804.

${ }_{33}$ Citado en Young-tsu Wong, op. cit., basándose en Boxer. Isaac Titsingh's Embassy to the Court of Ch ’ien Lung (1794-1795). "T’ien Hsia Monthly”, 8/1 (1931), pp. 9-33.

${ }_{34}$ Pierre Martial Cibot. Essai sur les jardins de plaisance des chinois [en Mémoires concernant l'histoire, les sciences, les artes, les. Vol. 8, Paris, Nyon, 1782], pp. 301-326.

35 Louis-François Delatour. Op. cit. 
La visita del Pachen Erdeni no ha podido reconstruirse plenamente, aunque parece posible que visitara los edificios. Mucho más clara es la visita del Pachen Lama, que resulta interesante en varios aspectos. Es conocido que se hospedó en una reproducción del palacio de los Pachen en Tashilhunpo levantada en el palacio de Chengde por indicación de Qiánlóng ${ }^{36}$. En este caso, no se optó por mostrar al visitante las construcciones exóticas de otras culturas. Se trataba de una visita de gran calado diplomático, en el que se mostraba precisamente la especial admiración que tenía el emperador chino por el budismo tibetano. Pero incluso en este caso, el emperador decidió enviar al Pachen Lama a visitar el Yuánmíng Yuán. De la descripción del jardín, previamente publicada, cabe destacar el pasaje dedicado a los edificios de gusto europeo.

Dans l'enceinte du palais appelé Khrus-'an il y avait des silhouettes animées, en bronze, représentant les douze années du cycle; chaucune était douée du pouvoir magique de faire tomber de l'eau de sa bouche à son propre moment. Sans comprendre, il les contempla attentivement, incapable du moindre commentaire $^{37}$.

Según han apuntado otros autores se estaría refiriendo a la fuente del Dàshǔrã 大水法 ${ }^{38}$. Aunque estas fueron importantes dentro del complejo, la indicación de esculturas de bronce hace más plausible que se esté refiriendo a la fuente del Hăiyàntáng 海晏堂 ${ }^{39}$. De allí, sería conducido al Făyuánlóu 法源楼 o Pabellón del Origen de la Doctrina ${ }^{40}$, donde permanecería dos días más. Incluso en este caso tan excepcional y cuidado diplomático, la visita a los palacios europeos resultó destacable. Sobre la embajada coreana es conocido que coincidieron con los holandeses en 1795 y que escribieron una descripción, que no ha podido localizarse. Aunque parece claro que estuvieron en el Yuánmíng Yuán, no queda especificado si visitaron el Xīyáng lóu.

Afortunadamente hay mucha más información sobre las descripciones europeas, aunque no todas son igual de accesibles. De reciente publicación es la de la embajada portuguesa, encabezada por Pacheco de Sampaio:

Passam depois à casa chamada europeia, assim classificada por Neuvialle por ser modernamente reedificada ao modo da Europa. O embaixador é mais explícito, dando aos padres a autoria do desenho, logo acrescentando, em jactância que já começa a maçar, que se havia concluído com a maior pressa, pelo grande

\footnotetext{
${ }^{36}$ James Hevia, op. Cit., p. 264.

${ }^{37}$ Anne Chayet. "Une description tibétaine du Yuanmingyuan". Michèle Pirazzoli-t'Serstevens. Le Yuanmingyuan...,p. 11.

${ }^{38}$ Ibid.

${ }^{39}$ Las cabezas de los animales de la fuente estaban realizadas en bronce. Se conservan siete de las doce, conocidas por haber sido subastadas en 2010.

${ }^{40}$ Parece probable que se hospedó en el pabellón de este nombre en el propio Yuánmíng Yuán, y no en el templo homónimo pekinés.
} 
empenho que o imperador teve que eu a visse na sua última perfeição. A fachada exterior não o terá impressionado. Diz apenas, de modo algo enigmático, que a naturalidade do arquiteto deve suprir a que falta na do edifício. Já sobre o interior é um pouco mais claro e liberal, contando que se achava a primeira sala ornada de muitas alfaias da Europa, a maior parte impróprias, mas todas estas impropriedades se reduziam a uma boa harmonia com a excelente pintura dos tectos, obra do grande Castiglione e de outros padres que servem no paço ${ }^{41}$.

Como ya se ha indicado en el estudio que publicó esta relación, se trata de la primera descripción de un visitante europeo a este sector, y además será la única de un delegado occidental hasta mucho después. Esta apertura podría interpretarse como una demostración de que el sector europeo no estuvo dedicado a los emisarios asiáticos antes que a los europeos. En cambio, las visitas en los años siguientes demuestran que tras esta excepción, el jardín quedó fuera del alcance de los visitantes occidentales. Así, la mucho más conocida embajada de Staunton y Macartney, desgrana su presencia en el jardín con muchos más detalles, pero sin incluir referencias a los edificios europeos.

At Pekin, the whole of the Embassy was lodged in a spacious edifice, or palace, consisting of several edifices, erected by a former collector of revenues and customs of Canton, out of his extortions, it was said, from the English trade, and confiscated to the crown [...]. This palace was built on the general model of the dwellings of great mandarins. The whole inclosure was in the form of a parallelogram, and surrounded by a high brick wall, the outside of which exhibited a plain blank surface, except near one of its angles, where a gateway opened into a narrow street, little promising the handsome structures within side ${ }^{42}$.

The only circumstance which militated against the picturesque in the landscape of the Chinese, was the formal shape and glaring colouring of their buildings. Their undulating roofs are, however, and exception to the first part of the charge; and their projection throws a softening shadow upon the colonnade which supports it. Some of those high towers, which Europeans call pagodas, are well adapted objects for vistas, and are accordingly, for the most part, placed on elevated situations ${ }^{43}$.

Between Haitien and Yuen-min-yuen, was the villa intended for the Embassador and his suite; which was an inclosure of, at least, twelve acres. It contained a garden laid out in serpentine walks, a rivulet winding round an island, a grove of various trees interspersed with patches of grass ground, and diversified with artificial inequalities, and rocks rudely heaped upon each other. The buildings in this place consisted of several separate pavilions, erected round small courts. The apartments were handsome, and not ill contrived. Several of them were adorned with landscapes, painted in water-colours ${ }^{44}$.

\footnotetext{
${ }^{41}$ António Vilhena de Carvalho. Nós os franceses mais quisemos ver toda a sua cabeça: dois relatos e uma embaixada a Pequim (Pacheco de Sampaio, 1753). "Oriente Ocidente", 32 (2015), pp. 42-52.

${ }^{42}$ George Staunton, op. Cit., p. 139.

${ }^{43}$ Ibid., op. Cit., p. 307.

${ }^{44}$ Ibid., p. 126.
} 
Una última descripción incluida en esta embajada, la de John Barrow, muestra que pudo tener una experiencia directa en el jardín, pero no cita los edificios europeos. El detalle con el que describe el resto lleva a pensar que no los vio.

Thirty distinct places of residence for the Emperor, with all the necessary appendages of building to each, for lodging the several officers to state [...]. These assemblages of buildings, which they dignify with the name of palaces, are, however, of such a nature as to be more remarkable for their number than for their splendour or magnificence. A great proportion of the buildings consists in mean cottages. [...] Their apartments are as deficient in proportion, as their construction is void of every rule and principle which we are apt to consider as essential to architecture. The principal hall of audience at Yuen-min-yuen stood upon a platform of granite, raised about four feet above the level of the court ${ }^{45}$.

El silencio sobre los edificios levantados por los misioneros europeos podría vincularse con una expedición concreta, pero ocurre lo mismo en la holandesa de 1795, cuyo historiador fue Van Braam ${ }^{46}$. Quedaron sorprendidos por los numerosos templos, construidos en las terrazas de las colinas, que superaban en belleza a los de la capital. Tuvieron la oportunidad de visitar el posteriormente conocido como Yíhéyuán 饾和园, y probablemente también el lago Kunming y la sala de audiencias. Curiosamente, en palabras del mismo Van Braam la expedición holandesa se arrepentía de no haber visitado los palacios europeos. Aunque solicitaron esta posibilidad a Héshēn 和珅, no fue atendida, lo que no comprendieron los enviados europeos.

Además de las embajadas, hubo otros europeos interesados en conocer e informar sobre las particularidades de estos edificios: los misioneros. Una de las primeras descripciones la ofrece el francés Attiret, que como residente en el jardín tuvo la capacidad de entrar y describirlo. No se trataba por tanto de un espacio vetado a los occidentales, sino a las misiones diplomáticas, quizás tras el poco éxito ante los portugueses.

Arrivé dans un vallon, on apperçoit les bâtimens. Toute la façade est en colomnes, \& en fenêtres: la charpente dorée, peinte, vernissée: les murailles de brique grise, bien taillée, bien polie: les toits sont couverts de tuiles vernissées, rouges, jaunes, bleues, vertes, violettes, qui par leur mélange \& leur arrangement font une agréable variété de compartimens \& de desseins. Ces bâtiments n'ont presque tous qu'un rez-de chaussée. Ils sont élevés de terre, de deux, quatre, six ou de huit pieds. [...]. Rien ne ressemble tant à ces Palais fabuleux de Fées, qu'on suppose au milieu d'un désert, élevés sur un roc dont l'avenue est raboteuse, $\&$ va en serpentant ${ }^{47}$.

${ }^{45}$ John Barrow, op. Cit., pp. 123-124.

${ }^{46}$ Young-tsu Wong, op. cit., 89-90.

47 "Lettre du Frere Attiret de la Compagnie de Jesus, peintre au service de l'Empereur de la Chine". Pekin, 1 de noviembre de 1743. Lettres edifiantes et curieuses, 27, Paris, Guerin, 1749, pp. 1-61 
Aussi leurs yeux accoûtumés à leur architecture, ne goûtent pas beaucoup notre maniere de bâtir. Voulez-vous sçavoir ce qu'ils en dissent, lorsqu'on leur en parle, ou qu'ils voient des estampes qui représentent nos bâtimens? Ces grands corps de logis, ces hauts pavillons les épouvantent; ils regardent nos rues comme des Chemins creusés dans d'affreuses montagnes, \& nos maisons comme des rochers à perte de vûe, percés de trous, ainsi que des habitations d'Ours \& d'autres bêtes féroces. Nos étages sur-tout, accumulés les uns sur les autres, leur paroissent insupportables; ils ne comprennent pas comment on peut risquer de se casser le col cent fois le jour, en montant nos degrés pour se render à un quatrième o cinquième étage. Il faut, disoit l'Empereur Cang-hy [Kangxi], en voyant les plans de nos maisons Européanes, il faut que l'Europe soit un pays bien petit \& bien miserable, puisqu'il n'y a pas affes de terrain pout étendre les villes, \& qu'on est oblige d'y habiter en l'air. Pour nous, nous concluons un peu différemment, $\&$ avec raison ${ }^{48}$.

Attiret no explica profundamente los edificios. Cibot podría haber sido una segunda fuente fundamental, pudiendo incorporar los edificios europeos a su historia del jardín chino. A pesar de trabajar en su construcción, decidió obviarlo. Un tercer misionero, Delatour describe finalmente los palacios con un detalle que no había sido conocido hasta el momento.

Vous jugerez mieux de ces maisons européennes bâties à Yuen-ming-yuen, par les XX grandes planches gravées qui les représentent, que je vous envoie. C'est le premier essai de gravure sur cuivre fait en Chine, sous les yeux et par les ordres de l'empereur. Ces maisons européennes n'ont que des ornements (prétendus) européens, pour en montrer le costume. Il est incroyable combien le souverain est riche en curiosités et en magnificences en tout genre, venues de l'Occident.

Vous me demandez si l'empereur a des glaces de Venise et de France; il y a plus de 30 années qu'il en avait déjà un si grand nombre, que ne sachant où les placer, il en fit couper une quantité de la première grandeur, pour faire des carreaux de croisées à ses bâtiments européens. Dans la salle qu'il a fait nouvellement bâtir pour placer les tapisseries de la manufacture des Gobelins, que la cour de France lui a envoyées en 1767, il y a partout des trumeaux magnifiques. Observez que cette salle, d'une dimension de 70 pieds de long, sur une belle largeur essais proportionnée, est si remplie de machines, qu'à peine trouve-ton au milieu un petit chemin pour passer: et telle de ces machines a coûté deux ou trois cent mille livres, parce que le travail en est exquis, et que les pierres précieuses dont on les a enrichies, sont en grand nombre.

$2^{\circ}$ Vous souhaitez savoir si les belles eaux jaillissantes du parc d'Yuen-mingyuen vont encore, et si, depuis le décès du père Benoist, nous avons des missionnaires en état de réparer les défauts des conduites, etc. Quand on se destinait à venir en Chine, on apprenait en particulier les arts qui pouvaient en ouvrir la porte et y être utiles; en sorte qu'il n'y a guère de missionnaire

48 Ibid., pp. 31-33. 
qui avec le secours des livres, n'ait assez d'avance pour faire ce qu'on peut lui demander. Ici, relativement aux choui-fa, ou jets et gerbes d'eau, etc., tout le monde s'en tirerait, mais c'est maintenant un talent inutile, du moins pour le moment. La machine qui fait monter les eaux dans le château d'eau (quoique formée avec bien des peines par le père Benoist), s'est à la vérité dérangée ou usée à la longue. On n'a pas cherché à la réparer, et les Chinois qui n'abandonnent que forcément leurs anciens usages, y sont revenus promptement, connaissant seulement pour tous travaux l'emploi des bras. C'est dans cette nation un système politique, d'employer et de faire vivre des gens dont la foule prodigieuse embarrasse, et dont l'oisiveté est dangereuse. Par exemple, on sait quand l'empereur doit aller se promener dans le quartier des bâtiments européens: un ou deux jours auparavant, on emploie tant de monde à porter l'eau que le bassin immense du château d'eau est suffisamment rempli, et les eaux jouent sur le passage de l'empereur ${ }^{49}$.

Además de esta visión general, que demuestra conocer también el envío de grabados, se hace eco de la descripción de cada plancha realizada por el Padre Avril ${ }^{50}$. En la misma muestra mucha información sobre las fuentes utilizadas por los misioneros a la hora de diseñar los palacios. La explicación se realiza siempre desde una perspectiva occidental, y no intentando incluir la visión china del plan.

Estas descripciones apuntan el uso de estos edificios. Algunos autores mantienen que se utilizaron para hospedar a diferentes miembros de la familia real o a cortesanos. Así parece desprenderse de hecho de algunos de los poemas sobre estos pabellones del hijo de Qiánlóng. Otros son más concretos vinculando la leyenda de la concubina uigur con los palacios europeos. En cambio, muchas fuentes y a partir de ellas autores como Kleughten prefieren considerarlos como meros contendores de obras de esos países lejanos ${ }^{51}$. De hecho, los misioneros que trabajaron en el jardín y se hospedaron allí, nunca lo hicieron en el entorno de los palacios europeos ${ }^{52}$.

Para finales del siglo XVIII se puede decir que los misioneros consiguieron acceder a los edificios del Xīyánglóu en calidad de artistas y responsables de las obras, pero esto no fue nada común entre las embajadas o viajeros. Una primera evidencia de la comparación entre estas descripciones es que ninguno de ellos llega a describir el jardín completo. Ni siquiera Attiret, que permaneció más libre y más tiempo en el recinto, parece que tuviera acceso a todos los espacios. La selección no se hizo por motivos de privacidad, dejando cerrados al público unos módulos concretos.

\footnotetext{
${ }^{49}$ Louis-François Delatour. Op. cit., pp. 126-127.

${ }^{50}$ Ibid., pp. 133-143.

${ }^{51}$ Kristina Kleughten, op. Cit., p. 181.

52 "La partie de ce bourg, dans laquelle notre maison Françoise a une petite résidence, pour y loger ceux des nôtres qui sont occupés à travailler dans le Palais de S. M. se nomme Hai tien". Lettres édifiantes et curieuses, 33, Paris, Berton, 1776, p. 180.
} 


\section{Representaciones gráficas del jardín en el siglo XVIII}

Qiánlóng mostró un temprano interés en representar pictóricamente el jardín. Este hecho facilitaría contar con una fuente más para interpretar las intenciones del emperador. La selección de las vistas y de los edificios, así como su orden dentro de los volúmenes puede utilizarse para valorar la función simbólica de los mismos. Lo que parece igualmente claro es que el emperador no realizó estos volúmenes con la intención de entregarlos como regalos diplomáticos a sus vasallos. Las copias se mantuvieron en Pekín, y solo salieron como fruto de regalos de los autores europeos a sus países, o como fruto del expolio. Hasta el momento no se ha hecho una revisión sistemática del número de copias y versiones realizadas durante el siglo XVIII del Yuánmíng Yuán. En la que se plantea a continuación se incluyen sólo aquellas que han intentado mostrar el jardín completo, o al menos, zonas importantes del mismo, obviando representaciones de edificios concretos, o detalles de los mismos incluidos en otras obras pictóricas.

En 1737, dos años después de iniciar su gobierno, el emperador solicitó a Giuseppe Castiglione, Tang Dai, Sun You, Shen Yuan, Zhang Wanban y Ding Guanpeng realizar un mapa del jardín ${ }^{53}$. El primer volumen dedicado a explicar el jardín bajo el reinado de Qiánlóng es el 圓明園四十景, o las Cuarenta vistas del Yuanmingyuan. escrito por el emperador, apoyado en dibujos de Tang Dai y Shen Yuan y la caligrafía de Wang Youdun, siendo editado en $1744^{54}$. Este mismo año es el de la finalización de las obras de los primeros palacios europeos, que no se incluyen entre las vistas. Castiglione estuvo en el equipo de trabajo, continuando su labor previa, por lo que debía conocer el aspecto de los edificios europeos, pero entre las vistas no se incluye ninguna de los palacios ${ }^{55}$. Podría pensarse que unas fechas tan ajustadas hacían imposible incorporarlos. De la misma forma, podría considerarse que desde el principio, el sector de estética europea formó un espacio interrelacionado con todo el jardín, pero independiente. Según Pirazzoli hay otra versión similar con veinte vistas en el 故宫博物院 Gugong Bowuyuan de Pekín ${ }^{56}$.

De este primer volumen existe una versión grabada desprovista de los poemas de Qiánlóng, titulado 中国皇帝的宫殿与庙宇 ${ }^{57}$. Esta obra fue realizada por Sun Hu y Shen Yuan en 1745, pasando a manos de Michel-Ferdinand d'Albert d'Ailly (1714-1769). Tras otros dueños, pasaría a su actual ubicación en la Biblioteca Nacional de Francia. De nuevo, no incluye los edificios

\footnotetext{
${ }^{53}$ Young-tsu Wong. Op. Cit., p. 25.

${ }^{54}$ Bibliothèque nationale de France, département Estampes et photographie, RESERVE FT 6-B-9.

${ }^{55}$ Victoria M. Siu. Op. Cit., p. 29.

${ }^{56}$ Michèle Pirazzoli-t’'Serstevems. Op. Cit., p. 10.

${ }^{57}$ Bibliothèque nationale de France, département Estampes et photographie, RESERVE PET FOLOE-21 (C)
} 
europeos, aunque podría interpretarse como una obra sacada directamente de la anterior, que no mereció incluir ninguna nueva incorporación.

Más tardío es el volumen 海淀: 中国皇帝的行宫, Haitien, El palacio de recreo del emperador de China ${ }^{58}$. Aquí también se han perdido los poemas del emperador, y tampoco se incorporan las vistas de los palacios europeos, pero se recupera el uso de las acuarelas. Además del volumen conservado en Paris, hay que destacar el ejemplar que Jean Joseph Grammont realizaría a finales del siglo XVIII y que se ha localizado en la Biblioteca del Palacio Real de Madrid ${ }^{59}$. Ese volumen es de menor calidad técnica y difiere en la interpretación de algunos detalles, por lo que parece claro que no son del mismo autor aunque debieron realizarse en paralelo. Aunque de nuevo podría explicarse como una versión del primer volumen, parece claro que si la inclusión de los palacios europeos hubiera sido considerada necesaria, se podría haber incluido.

Los palacios europeos fueron representados en una serie de veinte imágenes que se conservan en varias copias. Estos grabados fueron realizados por el pintor cortesano Ilantai, discípulo de Castiglione, en 1783 bajo el título Xiyanglou shuifa tu 西洋楼水法图 ${ }^{60}$. La versión manuscrita se encuentra en la Biblioteca Nacional de Francia ${ }^{61}$, donde también existe una versión grabada ${ }^{62}$. Otra de las series completas se encuentra en la Biblioteca Pública de Nueva York, aunque no se ha podido conocer su procedencia ${ }^{63}$. Una tercera se conserva en el Paço de San Miguel de Évora, aunque el número exacto de grabados conservados tampoco ha podido concretarse ${ }^{64}$. Más interés despierta la copia conservada en la Biblioteca de la Universidad de Manchester, que incluye una lámina coloreada de gran valor ${ }^{65}$. Otra versión incompleta forma parte

\footnotetext{
${ }^{58}$ Bibliothèque nationale de France, département Estampes et photographie, RESERVE PET FOL-OE-21.

${ }^{59}$ Yuan Ming Yuan. Palacio Real. Real Biblioteca. Grab/411.

${ }^{60}$ Kristina Kleugthen, op. cit.

${ }^{61}$ Vues de l'une des maisons de plaisances de l'Empereur de la Chine, à Yuen-ming-Yuen, construites dans le goût europeen. [Dessins de peintres chinois tracés en 1794 d'après les peintures originales des missionnaires jésuites.]. Bibliothèque nationale de France, département Fonds du service reproduction, PET FOL-OE-18.

${ }^{62}$ Esta versión ofrece la fecha exacta de edición, desconocida hasta ahora. Bibliothèque nationale de France, Richelieu, Département Estampes et photographie, OE-18(A)-FOL.

${ }^{63}$ A Suite of Twenty Engravings of the Yuan Ming-Yuan Summer Palaces and Gardens of the Chinese Emperor Ch'ien Lung. A Suite of Twenty Engravings of the Yuan Ming-Yuan Summer Palaces and Gardens of the Chinese Emperor Ch'ien Lung. A Suite of Twenty Engravings of the Yuan Ming-Yuan Summer Palaces and Gardens of the Chinese Emperor Ch'ien Lung. The Miriam and Ira D. Wallach Division of Art, Prints and Photographs: Print Collection, The New York Public LibraryThe Miriam and Ira D. Wallach Division of Art, Prints and Photographs: Print Collection, The New York Public LibraryThe Miriam and Ira D. Wallach Division of Art, Prints and Photographs: Print Collection, The New York Public LibraryThe Miriam and Ira D. Wallach Division of Art, Prints and Photographs: Print Collection, The New York Public LibraryThe Miriam and Ira D. Wallach Division of Art, Prints and Photographs: Print Collection, The New York Public Library.

${ }^{64}$ Por dificultades de acceso a los originales no se puede asegurar que se trate de un original del siglo XVIII.

${ }^{65}$ Twenty views of the European Palaces in the Garden of Perfect Brightness. Rylands Collection. Bookreader Chinese Collection 457.
} 
del Museo Británico ${ }^{66}$; mientras que otras siete vistas están fotografiadas en el Museo del Sudeste asiático de Estocolmo ${ }^{67}$. El ejemplar británico se conservó en el palacio de verano pekinés hasta el 19 de octubre de 1860 cuando fue obtenido por Henry Brooke. Por último, en el Museo Victoria \& Albert, se conserva otra copia casi completa ${ }^{68}$. Junto a todas estas, en los últimos años han salido al mercado en subastas internacionales algunas vistas sueltas, que pudieron formar parte de las series citadas, pero que apuntan a la existencia de al menos un volumen más ${ }^{69}$.

Esta serie de grabados, que sigue siendo la fuente principal para el estudio de estos edificios, está realizada desde el punto de vista occidental como ya apuntara Kleughten. Se insiste en las vistas frontales, aun siendo de laterales de los edificios, y no en cómo se presentan a lo largo de la experiencia del jardín. De la misma forma, la vista de pájaro, habitual en las otras obras presentadas, queda aquí desestimada. A partir del análisis de las representaciones conservadas parece claro que el jardín se describió por separado en su parte oriental y occidental, perdiendo el carácter unitario que debía tener el original. Las cortes europeas intentaron hacerse con ejemplares de las dos partes, quizás intentando tener fuentes suficientes para comprender el palacio.

\section{Arquitecturas exóticas y población local}

El acceso al recinto palaciego estuvo desde el origen muy controlado. Ni siquiera los príncipes, o los ministros del estado tenían habitualmente la posibilidad de visitarlo. Sólo se accedía por invitación, lo que solía ocurrir de forma excepcional para asistir a los espectáculos teatrales.

Qu'on observe encore que les princes du sang, les ministres d'État en Chine n'entrent point dans les jardins de l'empereur, et qu'il n'y a que ceux qui forment sa maison qui aient cette permission. Quelquefois ce prince y invite, soit pour la comédie, ou pour quelque autre spectacle, les princes du sang, les rois tributaires, etc, mais ils y sont conduits à l'endroit auquel ils sont invités, sans qu'on leur permette de s'écarter et d'aller voir d'autres parties des jardins ${ }^{70}$.

A esto había que unir que, en caso de visitarlo, se tuviera la oportunidad de conocer el sector europeo. En cualquier caso, era tiempo insuficiente para

\footnotetext{
${ }^{66}$ Cuenta con doce de las veinte vistas originales y los números de registro son de 1916,0214,01 a $1916,0214,0.4$ y de 1924,0523,0.13 a 1924,0523,0.21.

${ }^{67}$ Yuanmingyuan. 004517

${ }^{68}$ El albun conservado cuenta con 19 de las 20 vistas originales. Victoria \& Albert Museum, $\mathrm{n}^{\circ}$ 29452.

${ }^{69}$ Incluso habría que sumar las múltiples reediciones que ha tenido esta serie durante el siglo XX, que también han sido vendidas en algunos casos en subastas internacionales.

${ }^{70}$ Louis-François Delatour, op. Cit.
} 
tomar una información detallada de todas las estructuras. Cualquier impacto sobre las regiones más cercanas a Pekín debía realizarse por las imágenes que trasladaran los artistas residentes en el jardín. Desde esta perspectiva deben explicarse las representaciones de los palacios occidentales localizados recientemente por Hannibal Taubes en algunos puntos de Yuxian 蔚县 ${ }^{71}$. Se trata de unas representaciones de un gran interés, ya que no se pudieron realizar simplemente a partir del conocimiento de las estampas citadas previamente. Resulta muy probable que los artistas conocieran personalmente los palacios.

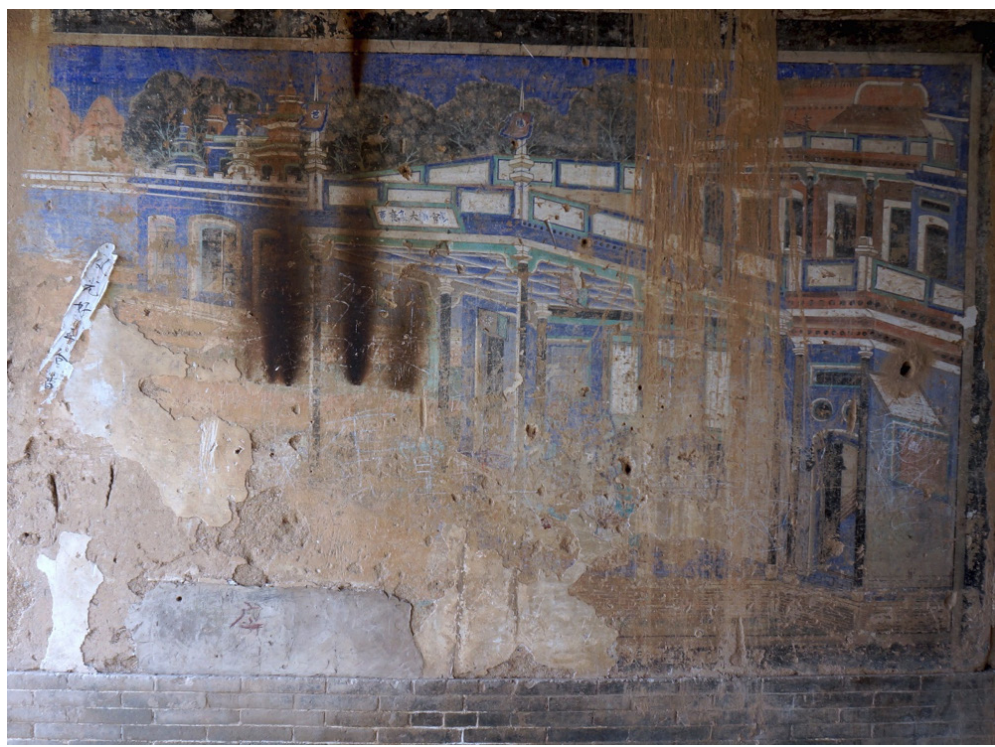

Fig. 4. China. Yuxian. Escenario de música. Pinturas murales.

Detalle. Fotografía de Hannibal Taubes

Esta hipótesis está basada en otros detalles como es la de los nombres de los edificios. Las pinturas citan expresamente la representación del 意大 宮, el Yìdà gōng (Fig. 4). Según los grabados no existe ningún edificio en el Yuánmíng Yuán con este nombre, y su significado en chino, el gran palacio de las intenciones, parece extraño. Taubes plantea como solución que el pintor resumiera el nombre real, 意大利宮, es decir, el palacio Yìdàlì o italiano, formulando una habitual transcripción fonética. Efectivamente, el edificio más claramente vinculado con la tradición italiana es el Hăiyàntáng 海晏堂, cuya escalera principal recuerda vagamente al palacio de Caprarola, en el que está basado según fuentes contemporáneas. La vista pintada en Yuxian no representa

\footnotetext{
${ }^{71}$ Queremos agradecer las aclaraciones realizadas gentilmente por Hannibal Taubes, de quien se espera un estudio sobre este particular en el futuro próximo.
} 
la fachada principal, ni ninguna de las laterales, aunque la parte alta es la misma que la del palacio. Una opción es que se trate del acceso al palacio desde el patio interior o desde la parte trasera.

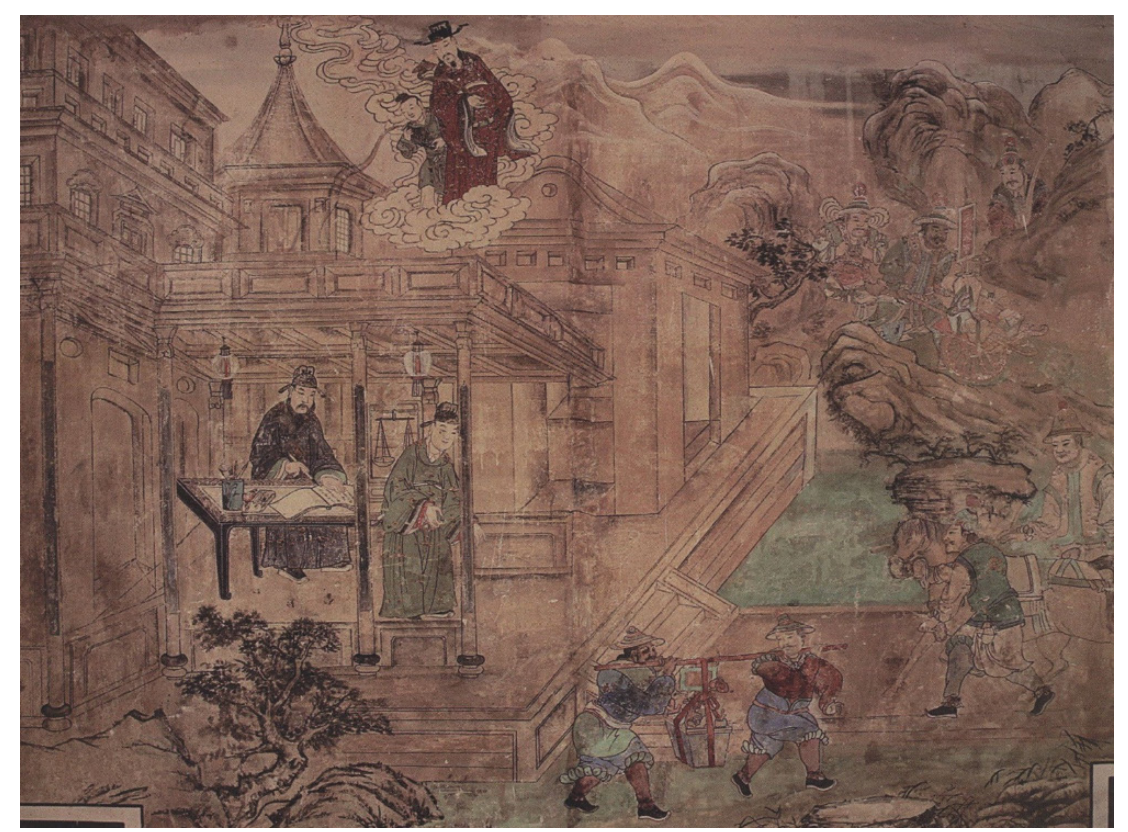

Fig. 5. China. Yuxian. Templo del Dios de la abundancia, 财神庙, incluyendo representaciones de los Xīyánglóu. Imagen publicada en 蔚縣博物館. 蔚 州寺廟壁畫. 北京: 科學出版社, 2013. Print. [Yu County Museum. Yu County Temple Frescoes. Beijing. Scientific Press. 2013. Print.].

Estos palacios no están representados exclusivamente por su rareza, sino por ser los escenarios de hechos maravillosos (Fig. 5). Las arquitecturas, necesariamente extrañas para el mundo chino, apoyaban la visión del mundo excepcional que viene apuntándose desde el principio de este trabajo. Pero además, las pinturas forman parte de la decoración de un escenario operístico o religioso. Aunque aún no se han localizado obras que se desarrollen específicamente en los jardines europeos, sí resulta claro que su selección para este espacio implica una vinculación con el mundo maravilloso reconocible por este público rural. 


\section{Conclusiones}

A partir de los elementos analizados en los apartados anteriores pueden plantearse algunas conclusiones finales. El Yuánmíng Yuán, aunque con estructuras previas a Qiánlóng, debe considerarse como una obra unitaria y con gran carga simbólica. Su función, aunque mantenía aspectos recreativos propios de emperadores anteriores, era claramente representativa. A pesar de funcionar como centro del poder imperial, fue un espacio poco visitado, con excepción de diplomáticos extranjeros y cortesanos en ocasiones significativas. De las descripciones se desprende que la visita de estos personajes no fue nunca completa, y que fue diseñada según su procedencia. Así los emisarios orientales habitualmente visitaron los edificios europeos, mientras que las occidentales, salvando alguna excepción, no lo pudieron hacer a pesar de su interés.

En el caso de las representaciones del jardín, resulta significativo que los palacios europeos no aparecen en las recopilaciones del Yuánmíng Yuán solicitadas por el emperador. Dicho esto, este hecho también puede explicarse por un problema de fechas. Sólo se conocen gracias a los grabados enviados por los misioneros a Europa. Esto podría llevar a pensar en que estos edificios formaban una especie de excepción marginal del jardín. Por el contrario, la reciente localización de pinturas de estos palacios en Yuxian hace pensar que entre aquellos chinos que conocieron estos espacios, los edificios contaban con un significado oriental pleno.

Todo esto demuestra que aunque las monarquías del siglo XVIII intentaron mostrarse ante sus cortes y delegaciones internacionales como poderes en contacto con los más distantes reinos, las diferencias entre ellas son destacables. Qiánlóng plantea una integración de la tradición europea dentro de la concepción budista, apostando por un modelo de universalidad propio mostrado a las misiones diplomáticas del momento. 


\section{Referencias bibliográficas:}

Maurice Adam, Yuen Ming Yuen. L'Oeuvre Architecturale des Anciens Jésuites au XVIIIe siècle, Pekin, Imprimerie des lazaristes, 1936.

Geremie R. Barmé. The Garden of Perfect Brightness, a life in ruins, "East Asian History", 11 (1996), pp. 111-158.

John Barrow. Travels in China. Londres, Cadell and W. Davies, 1804.

Schuyler Cammann. The Panchen Lama's Visit to China in 1780: An Episode in Anglo-Tibetan Relations. "The Far Eastern Quarterly”, 9/1 (1949), pp. 3-19.

Victoria M. Cha-Tsu Siu. Gardens of a Chinese Emperor. Imperial Creations of the Qianlong Era, 1736-1796. Bethlehem, Lehigh university press, 2013.

Che Bing Chiu. Un grand jardin imperial chinois: le Yuanming yuan, jardin de la Clarté parfait, "Extrême-Orient, Extrême-Occident", 22 (2000), pp. 17-50.

Pierre Martial Cibot. Essai sur les jardins de plaisance des chinois [en Mémoires concernant l'histoire, les sciences, les artes, les. Vol. 8, Paris, Nyon, 1782], pp. 301-326.

Daiheng Guo (ed.). 远逝的辉煌:圆明园建筑园林研究与保护. Shanghai: Shanghai keji jishu chubanshe, 2009.

圆明园的"记忆遗产" 样式房图档. Hangzhou: Zhejiang gu ji chu ban she, 2010.

Hope Danby, The Garden of Perfect Brightness: the history of the Yüan Ming Yüan and of the emperors who lived there, Chicago, Williams and Norgate, 1950.

Louis-François Delatour. Essais sur l'architecture des Chinois sur leurs jardins, leurs principes de médecine et leurs moeurs et usages. Paris, Clousier, 1803.

Benjamin A. Elman. The Jesuit Role as "Experts" in High Qing Cartography and Technology. “臺大歷史學報 National Taiwan University History Bulletin”, 31 (2003), pp. 223-250.

Alison Hardie, trans., The Craft of Gardens, New Haven y Londres, 1988.

John B. Henderson. Chinese Cosmographical Thought: The High Intellectual Tradition [en J. B. Harley and David Woodward (ed.), Cartography in the Traditional East and Southeast Asian Societies, Chicago, The University of Chicago Press, 1994], pp. 203-227.

James Hevia, Lamas, Emperors, and Rituals: Political Implications in Qing Imperial Ceremonies. "Journal of the International Association of Buddhist Studies”, 16/2 (1993), pp.

Hui Zou. The Jing of Line-Method: A perspective Garden in the Garden of Round Brightness. Montreal, McGill University, 2005. 
A Jesuit Garden in Beijing and Early Modern Chinese Culture. West Lafayette, Purdue University Press, 2011.

Kristina Kleutghen, Imperial Illusions: Crossing Pictorial Boundaries in the Qing Palaces, Seattle: University of Washington Press, 2015.

Cary Y. Liu. Architects and Builders of the Qing Dynasty Yuanming Yuan Imperial Garden-Palace, "The University of Hong Kong Museum Journal", 1 (2002), pp. 38-161.

Archive of Power. The Qing Dinasty Imperial Garden Palace at Rehe. “國立臺 灣大學美術史研究集刊”, 28 (2010), pp.. 43-82.

Liu Chang. From Study on Yuanming Yuan Interior Design to design Practice for the Beijing Chateau, Pekín, Tsinghua University, s.f.

Pedro Luengo. Intramuros, arquitectura en Manila 1739-1762. Madrid, Fundación Española de Universidades, 2012.

Arquitectura jesuita en Filipinas y China [en María Isabel Álvaro Zamora, Javier Ibáñez Fernández y Jesús Criado Mainar (coord.), La arquitectura jesuitica, Zaragoza, Institución Fernando el Católico, 2012], pp. 523-540. Identidad y globalización en las fachadas jesuitas de Pekín en el siglo XVIII' [en María Isabel Álvaro Zamora y Javier Ibáñez Fernández (coords.), La Compañía de Jesús y las artes: nuevas perspectivas de investigación, Zaragoza, Universidad de Zaragoza, 2014], pp. 279-300.

Manila, plaza fuerte. Ingenieros militares entre Europa, América y Asia. Madrid, Ministerio de Defensa, 2014.

James A. Millward, A Uyghur Muslim in Qianlong's Court. The Meaning of the Fragant Concubine, "The Journal of Asian Studies", 53/2 (1994), pp. 427-458.

Michèle Pirazzoli-t'Serstevens. Les Palais européens histoire et legends [en Michèle Pirazzoli-t'Serstevens (ed.), Le Yuanmingyuan. Jeux d'eau et palais européens du XVIIIe siècle à la cour de Chine, Paris, ERC, 1987], pp. 6-10.

Qingyu Jiang. Yuanming Yuan Garden: Space Arrangement Principles among Italy and China in Eighteenth Century. PhD Thesis. Politecnico di Torino, 2014.

George Staunton. Authentic Account of an Embassy from The King of Great Britain to the Emperor of China. Londres, W. Bulmer and Co., 1797.

Greg. M. Thomas. Yuanming Yuan/Versailles: Intercultural Interactions between Chinese and European Palace Cultures, "Art History", 32/1 (2009), pp. 115-143.

António Vilhena de Carvalho. Nós os franceses mais quisemos ver toda a sua cabeça: dois relatos e uma embaixada a Pequim (Pacheco de Sampaio, 1753). "Oriente Ocidente", 32 (2015), pp. 42-52. 
Xiangyun Wang, The Qing Cout's Tibet Connection: Lcang skya Rol pa'i rdo rje and the Qianlong Emperor. "Harvard Journal of Asiatic Studies", 60/1 (2000), pp. 125-163.

Young-tsu Wong. A Paradise lost: the imperial Garden Yuanming Yuan, Honolulu, University of Hawai'i Press, 2001.

Zheng Yangwen, An Unexplained Image from the Garden of Perfect Brightness, "China Heritage Quarterly”, 29 (2012).

China on the Sea. How the Maritime World Shaped Modern China, Leiden, Brill, 2012. 